\title{
SUBELLIPTIC AND PARAMETRIC EQUATIONS ON CARNOT GROUPS
}

\author{
GIOVANNI MOLICA BISCI AND MASSIMILIANO FERRARA
}

(Communicated by Svitlana Mayboroda)

\begin{abstract}
This article concerns a class of elliptic equations on Carnot groups depending on one real parameter. Our approach is based on variational methods. More precisely, we establish the existence of at least two weak solutions for the treated problem by using a direct consequence of the celebrated PucciSerrin theorem and of a local minimum result for differentiable functionals due to Ricceri.
\end{abstract}

\section{INTRODUCTION}

It is well known that a great deal of attention has been focused by many authors on the study of subelliptic equations on Carnot groups and in particular on the Heisenberg group $\mathbb{H}^{n}$. See, among others, the papers [2, 4, 8, 10, 12] and references therein.

Motivated by this large interest in the literature, we study here the existence of weak solutions for the following problem

$$
\left(P_{\lambda}^{f}\right) \quad\left\{\begin{array}{l}
-\Delta_{\mathbb{G}} u=\lambda f(\xi, u) \quad \text { in } D \\
\left.u\right|_{\partial D}=0,
\end{array}\right.
$$

where $D$ is a smooth bounded domain of the Carnot group $\mathbb{G}, \Delta_{\mathbb{G}}$ is the subelliptic Laplacian on $\mathbb{G}$, and $\lambda$ is a positive real parameter.

More precisely, inspired by [1] and 14, we prove that, for small values of $\lambda$, problem $\left(P_{\lambda}^{f}\right)$ admits at least two weak solutions requiring that the continuous and subcritical nonlinear term $f$ satisfies the celebrated Ambrosetti-Rabinowitz condition without the usual additional assumption at zero, that is,

$$
\lim _{t \rightarrow 0} \frac{f(\xi, t)}{t}=0
$$

uniformly in $D$.

Received by the editors May 10, 2015 and, in revised form, August 17, 2015 and September 3, 2015 .

2010 Mathematics Subject Classification. Primary 35J65; Secondary 22E25.

Key words and phrases. Subelliptic equations, Carnot groups, multiple solutions, critical point results. 
A special case of our result reads as follows.

Theorem 1.1. Let $D$ be a smooth and bounded domain of the Heisenberg group $\mathbb{H}^{n}$ and $f: \mathbb{R} \rightarrow \mathbb{R}$ be a continuous function for which

$\left(f_{1}^{\prime}\right)$ there exist $a_{1}, a_{2}>0$ and $q \in\left(2,2\left(\frac{n+1}{n}\right)\right)$ such that

$$
|f(t)| \leq a_{1}+a_{2}|t|^{q-1}
$$

for every $t \in \mathbb{R}$;

$\left(f_{2}^{\prime}\right)$ there are $\alpha>2$ and $r_{0}>0$ such that

$$
0<\alpha \int_{0}^{t} f(\tau) d \tau \leq t f(t)
$$

for any $|t| \geq r_{0}$.

Then, there exists an open interval $\Lambda \subset(0,+\infty)$ such that, for every $\lambda \in \Lambda$, the following problem

$$
\left\{\begin{array}{l}
-\Delta_{\mathbb{H}^{n}} u=\lambda f(u) \quad \text { in } D \\
\left.u\right|_{\partial D}=0,
\end{array}\right.
$$

admits at least two (distinct) weak solutions in the Folland-Stein space $S_{0}^{1}(D)$.

The interval $\Lambda$ in the above result can be explicitly determined. Precisely, setting

$$
c_{s}:=\sup _{u \in S_{0}^{1}(D) \backslash\{0\}} \frac{\|u\|_{L^{s}(D)}}{\|u\|_{S_{0}^{1}(D)}}, \quad(\text { with } s \in\{1, q\}),
$$

one has

$$
\Lambda:=\left(0, \frac{q}{2} \max _{\varrho>0} \frac{\sqrt{\varrho}}{a_{1} c_{1} q+a_{2} c_{q}^{q} \varrho^{\frac{q-1}{2}}}\right) ;
$$

see Remark 4.2 for details.

Our abstract tool for proving the main result is the following abstract theorem that we recall here in a convenient form.

Theorem 1.2. Let $E$ be a reflexive real Banach space, and let $\Phi, \Psi: E \rightarrow \mathbb{R}$ be two continuously Gâteaux differentiable functionals such that $\Phi$ is sequentially weakly lower semicontinuous and coercive. Further, assume that $\Psi$ is sequentially weakly continuous. In addition, assume that, for each $\mu>0$, the functional $J_{\mu}:=\mu \Phi-\Psi$ satisfies the classical compactness Palais-Smale (briefly (PS)) condition. Then, for each $\varrho>\inf _{E} \Phi$ and each

$$
\mu>\inf _{u \in \Phi^{-1}((-\infty, \varrho))} \frac{\sup _{v \in \Phi^{-1}((-\infty, \varrho))} \Psi(v)-\Psi(u)}{\varrho-\Phi(u)},
$$

the following alternative holds: either the functional $J_{\mu}$ has a strict global minimum which lies in $\Phi^{-1}((-\infty, \varrho))$, or $J_{\mu}$ has at least two critical points one of which lies in $\Phi^{-1}((-\infty, \varrho))$.

The above critical point result comes from a joint application of the classical Pucci-Serrin theorem (see [13]) and a local minimum result due to Ricceri (see [15]). For a proof of Theorem [1.2 see, for instance, 14, Theorem 6]. We refer the interested reader to [11,16, 17] and references therein for recent applications of Ricceri's variational principle. 
The plan of the paper is as follows. Section 2 is devoted to our abstract framework and preliminaries. Successively, in Section 3 . Theorem 3.1 and some preparatory results concerning the compactness Palais-Smale condition (see Lemmas 3.3 and 3.4) are presented.

In the last section, Theorem 3.1 is proved, and a concrete example of an application is presented in Example 4.3 .

\section{Abstract FrameWork}

In this section we briefly recall some basic facts on Carnot groups and the functional space $S_{0}^{1}(D)$. Let $\left(\mathbb{R}^{n}\right.$, o) be a Lie Group equipped with a family of group automorphisms, namely dilatations, $\mathfrak{F}:=\left\{\delta_{\eta}\right\}_{\eta>0}$ such that, for every $\eta>0$, the map

$$
\delta_{\eta}: \prod_{k=1}^{r} \mathbb{R}^{n_{k}} \rightarrow \prod_{k=1}^{r} \mathbb{R}^{n_{k}}
$$

is given by

$$
\delta_{\eta}\left(\xi^{(1)}, \ldots, \xi^{(r)}\right):=\left(\eta \xi^{(1)}, \eta^{2} \xi^{(2)}, \ldots, \eta^{r} \xi^{(r)}\right)
$$

where $\xi^{(k)} \in \mathbb{R}^{n_{k}}$ for every $k \in\{1, \ldots, r\}$ and $\sum_{k=1}^{r} n_{k}=n$.

The structure $\mathbb{G}:=\left(\mathbb{R}^{n}, \circ, \mathfrak{F}\right)$ is called a homogeneous group with homogeneous dimension

$$
\operatorname{dim}_{h} \mathbb{G}:=\sum_{k=1}^{r} k n_{k} .
$$

From now on, we shall assume $\operatorname{dim}_{h} \mathbb{G} \geq 3$. We remark that, if $\operatorname{dim}_{h} \mathbb{G} \leq 3$, then necessarily $\mathbb{G}=\left(\mathbb{R}^{\operatorname{dim}_{h} \mathbb{G}},+\right)$. Note that the number $\operatorname{dim}_{h} \mathbb{G}$ is naturally associated to the family $\mathfrak{F}$ since, for every $\eta>0$, the Jacobian of the map

$$
\xi \mapsto \delta_{\eta}(\xi), \quad \forall \xi \in \mathbb{R}^{n}
$$

equals $\eta^{\operatorname{dim}_{h} \mathbb{G}}$. Now, let $\mathfrak{g}$ be the Lie algebra of left invariant vector fields on $\mathbb{G}$ and assume that $\mathfrak{g}$ is stratified, that is,

$$
\mathfrak{g}=\bigoplus_{k=1}^{r} V_{k}
$$

where the integer $r$ is called the step of $\mathbb{G}, V_{k}$ is a linear subspace of $\mathfrak{g}$, for every $k \in\{1, \ldots, r\}$, and

$$
\begin{aligned}
& \operatorname{dim} V_{k}=n_{k}, \text { for every } k \in\{1, \ldots, r\}, \\
& {\left[V_{1}, V_{k}\right]=V_{k+1}, \text { for } 1 \leq k \leq r-1, \text { and }\left[V_{1}, V_{r}\right]=\{0\} .}
\end{aligned}
$$

In this setting the symbol $\left[V_{1}, V_{k}\right]$ denotes the subspace of $\mathfrak{g}$ generated by the commutators $[X, Y]$, where $X \in V_{1}$ and $Y \in V_{k}$.

A Carnot group is a homogeneous group $\mathbb{G}$ such that the Lie algebra $\mathfrak{g}$, associated to $\mathbb{G}$, is stratified. Moreover, the subelliptic Laplacian operator on $\mathbb{G}$ is the secondorder differential operator, given by

$$
\Delta_{\mathbb{G}}:=\sum_{k=1}^{n_{1}} X_{k}^{2}
$$


where $\left\{X_{1}, \ldots, X_{n_{1}}\right\}$ is a basis of $V_{1}$. We shall denote by

$$
\nabla_{\mathbb{G}}:=\left(X_{1}, \ldots, X_{n_{1}}\right)
$$

the related horizontal gradient.

A crucial role in the functional analysis on Carnot groups is played by the following Sobolev-type inequality:

$$
\int_{D}|u(\xi)|^{2^{*}} d \xi \leq C \int_{D}\left|\nabla_{\mathbb{G}} u(\xi)\right|^{2} d \xi, \quad \forall u \in C_{0}^{\infty}(D),
$$

due to Folland (see [6]). In the above expression $C$ is a positive constant (independent on $u$ ) and

$$
2^{*}:=\frac{2 \operatorname{dim}_{h} \mathbb{G}}{\operatorname{dim}_{h} \mathbb{G}-2}
$$

is the critical Sobolev exponent. Inequality (3) ensures that if $D$ is a bounded open (smooth) subset of $\mathbb{G}$, then the function

$$
u \mapsto\|u\|_{S_{0}^{1}(D)}:=\left(\int_{D}\left|\nabla_{\mathbb{G}} u(\xi)\right|^{2} d \xi\right)^{1 / 2}
$$

is a norm in $C_{0}^{\infty}(D)$.

We shall denote by $S_{0}^{1}(D)$ the Folland-Stein space defined as the completion of $C_{0}^{\infty}(D)$ with respect to the norm $\|\cdot\|_{S_{0}^{1}(D)}$. The exponent $2^{*}$ is critical for $\Delta_{\mathbb{G}}$ since, as in the classical Laplacian setting, the embedding $S_{0}^{1}(D) \hookrightarrow L^{q}(D)$ is compact when $1 \leq q<2^{*}$, while it is only continuous if $q=2^{*}$ (see Folland and Stein [7]).

The simplest example of a Carnot group is provided by the Heisenberg group $\mathbb{H}^{n}:=\left(\mathbb{R}^{2 n+1}, \circ\right)$, where, for every

$$
p:=\left(p_{1}, \ldots, p_{2 n}, p_{2 n+1}\right) \text { and } q:=\left(q_{1}, \ldots, q_{2 n}, q_{2 n+1}\right) \in \mathbb{H}^{n},
$$

the usual group operation $\circ: \mathbb{H}^{n} \times \mathbb{H}^{n} \rightarrow \mathbb{H}^{n}$ is given by

$$
p \circ q:=\left(p_{1}+q_{1}, \ldots, p_{2 n}+q_{2 n}, p_{2 n+1}+q_{2 n+1}+\frac{1}{2} \sum_{k=1}^{2 n}\left(p_{k} q_{k+n}-p_{k+n} q_{k}\right)\right),
$$

and the family of dilatations has the following form:

$$
\delta_{\eta}(p):=\left(\eta p_{1}, \ldots, \eta p_{2 n}, \eta^{2} p_{2 n+1}\right), \quad \forall \eta>0 .
$$

Thus, by (2) it follows that

$$
\operatorname{dim}_{h} \mathbb{H}^{n}:=2 n+2 .
$$

The Lie algebra of left invariant vector fields on $\mathbb{H}^{n}$ is denoted by $\mathfrak{h}$ and its standard basis is given by

$$
\begin{gathered}
X_{k}:=\partial_{k}-\frac{p_{n+k}}{2} \partial_{2 n+1}, \quad k \in\{1, \ldots, n\}, \\
Y_{k}:=\partial_{n+k}-\frac{p_{k}}{2} \partial_{2 n+1}, \quad k \in\{1, \ldots, n\}, \\
T:=\partial_{2 n+1} .
\end{gathered}
$$

In such a case, the only nontrivial commutator relations are

$$
\left[X_{k}, Y_{k}\right]=T, \quad \forall k \in\{1, \ldots, n\} .
$$

Finally, the stratification of $\mathfrak{h}$ is given by

$$
\mathfrak{h}=\operatorname{span}\left\{X_{1}, \ldots, X_{n}, Y_{1}, \ldots, Y_{n}\right\} \oplus \operatorname{span}\{T\} .
$$


We cite the monograph [3] for a nice introduction on Carnot groups and 9] for related topics on variational methods used in this paper.

\section{The MAIN RESUlt AND SOME TECHNICAL LEMMAS}

The aim of this section is to prove that, under natural assumptions on the nonlinear term $f$, weak solutions to problem $\left(P_{\lambda}^{f}\right)$ below do exist. With the above notation the main result reads as follows.

Theorem 3.1. Let $D$ be a smooth and bounded domain of a Carnot group $\mathbb{G}$ with $\operatorname{dim}_{h} \mathbb{G} \geq 3$, and $f: \bar{D} \times \mathbb{R} \rightarrow \mathbb{R}$ be a continuous function such that

$\left(f_{1}\right)$ there exist $a_{1}, a_{2}>0$ and $q \in\left(2,2^{*}\right)$ such that

$$
|f(\xi, t)| \leq a_{1}+a_{2}|t|^{q-1}
$$

for every $\xi \in D$ and $t \in \mathbb{R}$

$\left(f_{2}\right)$ there are $\alpha>2$ and $r_{0}>0$ such that

$$
0<\alpha \int_{0}^{t} f(\xi, \tau) d \tau \leq t f(\xi, t)
$$

for any $\xi \in \bar{D}$, and $|t| \geq r_{0}$.

Then, for every $\varrho>0$ and each

$$
0<\lambda<\frac{q \sqrt{\varrho}}{2\left(a_{1} c_{1} q+a_{2} c_{q}^{q} \varrho^{\frac{q-1}{2}}\right)},
$$

problem $\left(P_{\lambda}^{f}\right)$ admits at least two weak solutions one of which lies in

$$
\mathbb{B}_{\varrho}:=\left\{u \in S_{0}^{1}(D):\|u\|_{S_{0}^{1}(D)}^{2}<\varrho\right\} .
$$

We recall that a weak solution for the problem $\left(P_{\lambda}^{f}\right)$ is a function $u: D \rightarrow \mathbb{R}$ such that

$$
\left\{\begin{array}{l}
\int_{D}\left\langle\nabla_{\mathbb{G}} u(\xi), \nabla_{\mathbb{G}} \varphi(\xi)\right\rangle d \xi \\
u \in S_{0}^{1}(D) .
\end{array}=\lambda \int_{D} f(\xi, u(\xi)) \varphi(\xi) d \xi, \quad \forall \varphi \in S_{0}^{1}(D),\right.
$$

Remark 3.2. Assuming the Ambrosetti-Rabinowitz condition $\left(f_{2}\right)$, standard computations ensure that the potential $F(\xi, t):=\int_{0}^{t} f(\xi, \tau) d \tau$ is $\alpha$-superhomogeneous at infinity, i.e.,

$$
F(\xi, t v) \geq F(\xi, v) t^{\alpha}
$$

for every $\xi \in \bar{D},(t, v) \in \mathbb{R}^{2}$ with $t \geq 1$ and $|v| \geq r_{0}$. Indeed, for $t=1$, clearly the equality holds. Otherwise, fix $|v| \geq r_{0}$ and define $g(\xi, t):=F(\xi, t v)$, for every $x \in \bar{D}$ and $t \in(1,+\infty)$. By $\left(f_{2}\right)$ it follows that

$$
\frac{g^{\prime}(\xi, t)}{g(\xi, t)} \geq \frac{\alpha}{t}
$$

for every $\xi \in \bar{D}$ and $t>1$. By integrating in $(1, t]$ we get that

$$
\int_{1}^{t} \frac{g^{\prime}(\xi, s)}{g(\xi, s)} d s=\log \frac{g(\xi, t)}{g(\xi, 1)} \geq \log t^{\alpha} .
$$


In conclusion, the claim is verified since one has

$$
\begin{aligned}
F(\xi, t v) & =: g(\xi, t) \\
& \geq g(\xi, 1) t^{\alpha} \\
& =F(\xi, v) t^{\alpha}
\end{aligned}
$$

for every $\xi \in \bar{D},|v| \geq r_{0}$ and $t>1$.

Now, for the sake of completeness, we recall that a $C^{1}$-functional $J: E \rightarrow \mathbb{R}$, where $E$ is a real Banach space with topological dual $E^{*}$, satisfies the Palais-Smale condition at level $\zeta \in \mathbb{R},\left(\right.$ abbreviated $\left.(\mathrm{PS})_{\zeta}\right)$ when

$(\mathrm{PS})_{\zeta}$ Every sequence $\left\{u_{j}\right\}_{j \in \mathbb{N}}$ in $E$ such that

$$
J\left(u_{j}\right) \rightarrow \zeta, \quad \text { and }\left\|J^{\prime}\left(u_{j}\right)\right\|_{E^{*}} \rightarrow 0,
$$

as $j \rightarrow+\infty$, possesses a convergent subsequence.

We say that $J$ satisfies the Palais-Smale condition (abbreviated (PS)) if $(\mathrm{PS})_{\zeta}$ holds for every $\zeta \in \mathbb{R}$.

For our goal, in the next two lemmas we shall verify the compactness (PS) condition for the functional $\mathcal{J}_{\lambda}: S_{0}^{1}(D) \rightarrow \mathbb{R}$ defined by

$$
\mathcal{J}_{\lambda}(u):=\frac{1}{2 \lambda}\|u\|_{S_{0}^{1}(D)}^{2}-\int_{D} F(\xi, u(\xi)) d \xi, \quad \forall u \in S_{0}^{1}(D),
$$

where $\lambda \in \mathbb{R}$ and, as usual, we set $F(\xi, t):=\int_{0}^{t} f(\xi, \tau) d \tau$.

Note that the functional $\mathcal{J}_{\lambda} \in C^{1}\left(S_{0}^{1}(D)\right)$ and its derivative at $u \in S_{0}^{1}(D)$ is given by

$$
\begin{aligned}
\left\langle\mathcal{J}_{\lambda}^{\prime}(u), \varphi\right\rangle=\frac{1}{\lambda} \int_{D}\left\langle\nabla_{\mathbb{G}} u(\xi),\right. & \left.\nabla_{\mathbb{G}} \varphi(\xi)\right\rangle d \xi \\
& -\int_{D} f(\xi, u(\xi)) \varphi(\xi) d \xi
\end{aligned}
$$

for every $\varphi \in S_{0}^{1}(D)$.

Lemma 3.3. Assume that conditions $\left(f_{1}\right)$ and $\left(f_{2}\right)$ are verified. Then, every Palais-Smale sequence for the functional $\mathcal{J}_{\lambda}$ is bounded in $S_{0}^{1}(D)$.

Proof. Let $\left\{u_{j}\right\}_{j \in \mathbb{N}} \subset S_{0}^{1}(D)$ be a Palais-Smale sequence, i.e.,

$$
\mathcal{J}_{\lambda}\left(u_{j}\right) \rightarrow \zeta
$$

for $\zeta \in \mathbb{R}$ and

$$
\left\|\mathcal{J}_{\lambda}^{\prime}\left(u_{j}\right)\right\|_{S^{-1}} \rightarrow 0
$$

as $j \rightarrow \infty$, where

$$
\left\|\mathcal{J}_{\lambda}^{\prime}\left(u_{j}\right)\right\|_{S^{-1}}:=\sup \left\{\left|\left\langle\mathcal{J}_{\lambda}^{\prime}\left(u_{j}\right), \varphi\right\rangle\right|: \varphi \in S_{0}^{1}(D),\|\varphi\|_{S_{0}^{1}(D)}=1\right\} .
$$

We argue by contradiction. So, suppose to the contrary that the conclusion were not true. Passing to a subsequence if necessary, we may assume that

$$
\left\|u_{j}\right\|_{S_{0}^{1}(D)} \rightarrow+\infty
$$


as $j \rightarrow+\infty$. It follows that there exists $j_{0} \in \mathbb{N}$ such that

$$
\begin{aligned}
\mathcal{J}_{\lambda}\left(u_{j}\right)- & \frac{\left\langle\mathcal{J}_{\lambda}^{\prime}\left(u_{j}\right), u_{j}\right\rangle}{\alpha} \\
= & \frac{1}{2 \lambda}\left(\frac{\alpha-2}{\alpha}\right)\left\|u_{j}\right\|_{S_{0}^{1}(D)}^{2} \\
& +\int_{D}\left[\frac{f\left(\xi, u_{j}(\xi)\right) u_{j}(\xi)}{\alpha}-F\left(\xi, u_{j}(\xi)\right)\right] d \xi
\end{aligned}
$$

for every $j \geq j_{0}$.

Thus

$$
\begin{aligned}
\frac{1}{2 \lambda}\left(\frac{\alpha-2}{\alpha}\right)\left\|u_{j}\right\|_{S_{0}^{1}(D)}^{2} & \leq \mathcal{J}_{\lambda}\left(u_{j}\right)-\frac{\left\langle\mathcal{J}_{\lambda}^{\prime}\left(u_{j}\right), u_{j}\right\rangle}{\alpha} \\
& -\int_{\left|u_{j}(\xi)\right|>r_{0}}\left[\frac{f\left(\xi, u_{j}(\xi)\right) u_{j}(\xi)}{\alpha}-F\left(\xi, u_{j}(\xi)\right)\right] d \xi \\
& +\kappa_{1}|D|, \quad \forall j \geq j_{0},
\end{aligned}
$$

where $|D|$ denotes the measure of $D$ and

$$
\kappa_{1}:=\sup \left\{\left|\frac{f(\xi, t) t}{\alpha}-F(\xi, t)\right|: \xi \in \bar{D},|t| \leq r_{0}\right\} .
$$

Now, we observe that condition $\left(f_{2}\right)$ yields

$$
\int_{\left|u_{j}(\xi)\right|>r_{0}}\left[\frac{f\left(\xi, u_{j}(\xi)\right) u_{j}(\xi)}{\alpha}-F\left(\xi, u_{j}(\xi)\right)\right] d \xi \geq 0 .
$$

So, we deduce that

$$
\frac{1}{2 \lambda}\left(\frac{\alpha-2}{\alpha}\right)\left\|u_{j}\right\|_{S_{0}^{1}(D)}^{2} \leq \mathcal{J}_{\lambda}\left(u_{j}\right)-\frac{\left\langle\mathcal{J}_{\lambda}^{\prime}\left(u_{j}\right), u_{j}\right\rangle}{\alpha}+\kappa_{1}|D|,
$$

for every $j \geq j_{0}$. Then, for every $j \geq j_{0}$ one has

$$
\frac{1}{2 \lambda}\left(\frac{\alpha-2}{\alpha}\right)\left\|u_{j}\right\|_{S_{0}^{1}(D)}^{2} \leq \mathcal{J}_{\lambda}\left(u_{j}\right)+\frac{1}{\alpha}\left\|\mathcal{J}_{\lambda}^{\prime}\left(u_{j}\right)\right\|_{S^{-1}}\left\|u_{j}\right\|_{S_{0}^{1}(D)}+\kappa_{1}|D| .
$$

In conclusion, dividing by $\left\|u_{j}\right\|_{S_{0}^{1}(D)}$ and letting $j \rightarrow+\infty$, we obtain a contradiction.

Lemma 3.4. Assume that conditions $\left(f_{1}\right)$ and $\left(f_{2}\right)$ are verified. Then, the functional $\mathcal{J}_{\lambda}$ satisfies the compactness (PS) condition.

Proof. Let $\left\{u_{j}\right\}_{j \in \mathbb{N}} \subset S_{0}^{1}(D)$ be a Palais-Smale sequence. By Lemma 3.3, the sequence $\left\{u_{j}\right\}_{j \in \mathbb{N}}$ is necessarily bounded in $S_{0}^{1}(D)$. Since $S_{0}^{1}(D)$ is reflexive, we can extract a subsequence which for simplicity we shall call again $\left\{u_{j}\right\}_{j \in \mathbb{N}}$, such that $u_{j} \rightarrow u_{\infty}$ in $S_{0}^{1}(D)$. This means that

$$
\begin{aligned}
& \int_{D}\left\langle\nabla_{\mathbb{G}} u_{j}(\xi), \nabla_{\mathbb{G}} \varphi(\xi)\right\rangle d \xi \rightarrow \\
& \int_{D}\left\langle\nabla_{\mathbb{G}} u_{\infty}(\xi), \nabla_{\mathbb{G}} \varphi(\xi)\right\rangle d \xi
\end{aligned}
$$

for any $\varphi \in S_{0}^{1}(D)$, as $j \rightarrow+\infty$. 
We will prove that $u_{j}$ strongly converges to $u_{\infty} \in S_{0}^{1}(D)$. Exploiting the derivative $\mathcal{J}_{\lambda}^{\prime}\left(u_{j}\right)\left(u_{j}-u_{\infty}\right)$, we obtain

$$
\begin{aligned}
\left\langle a\left(u_{j}\right), u_{j}-u_{\infty}\right\rangle= & \left\langle\mathcal{J}_{\lambda}^{\prime}\left(u_{j}\right), u_{j}-u_{\infty}\right\rangle \\
& +\int_{D} f\left(\xi, u_{j}(\xi)\right)\left(u_{j}-u_{\infty}\right)(\xi) d \xi
\end{aligned}
$$

where we set

$$
\left\langle a\left(u_{j}\right), u_{j}-u_{\infty}\right\rangle:=\frac{1}{\lambda}\left(\left\|u_{j}\right\|_{S_{0}^{1}(D)}^{2}-\int_{D}\left\langle\nabla_{\mathbb{G}} u_{j}(\xi), \nabla_{\mathbb{G}} u_{\infty}(\xi)\right\rangle d \xi\right) .
$$

Since $\left\|\mathcal{J}_{\lambda}^{\prime}\left(u_{j}\right)\right\|_{S^{-1}} \rightarrow 0$ and (by Lemma 3.3) the sequence $\left\{u_{j}-u_{\infty}\right\}_{j \in \mathbb{N}}$ is bounded in $S_{0}^{1}(D)$, taking into account that

$$
\left|\left\langle\mathcal{J}_{\lambda}^{\prime}\left(u_{j}\right), u_{j}-u_{\infty}\right\rangle\right| \leq\left\|\mathcal{J}_{\lambda}^{\prime}\left(u_{j}\right)\right\|_{S^{-1}}\left\|u_{j}-u_{\infty}\right\|_{S_{0}^{1}(D)},
$$

one gets

$$
\left\langle\mathcal{J}_{\lambda}^{\prime}\left(u_{j}\right), u_{j}-u_{\infty}\right\rangle \rightarrow 0
$$

as $j \rightarrow+\infty$.

Now, since the embedding $S_{0}^{1}(D) \hookrightarrow L^{q}(D)$ is compact, clearly $u_{j} \rightarrow u_{\infty}$ strongly in $L^{q}(D)$. So, by condition $\left(f_{1}\right)$, we obtain

$$
\int_{D}\left|f\left(\xi, u_{j}(\xi)\right)\right|\left|u_{j} \|(\xi)-u_{\infty}(\xi)\right| d \xi \rightarrow 0 .
$$

By (10) relations (11) and (12) yield

$$
\left\langle a\left(u_{j}\right), u_{j}-u_{\infty}\right\rangle \rightarrow 0
$$

when $j \rightarrow+\infty$.

Hence by (13) we can write

$$
\int_{D}\left|\nabla_{\mathbb{G}} u_{j}(\xi)\right|^{2} d \xi-\int_{D}\left\langle\nabla_{\mathbb{G}} u_{j}(\xi), \nabla_{\mathbb{G}} u_{\infty}(\xi)\right\rangle d \xi \rightarrow 0,
$$

when $j \rightarrow+\infty$.

Thus, by (14) and (9) it follows that

$$
\limsup _{j \rightarrow \infty} \int_{D}\left|\nabla_{\mathbb{G}} u_{j}(\xi)\right|^{2} d \xi=\int_{D}\left|\nabla_{\mathbb{G}} u_{\infty}(\xi)\right|^{2} d \xi
$$

In conclusion, thanks to [5, Proposition III.30], $u_{j} \rightarrow u_{\infty}$ in $S_{0}^{1}(D)$. The proof is thus complete.

\section{Proof of Theorem 3.1}

For the proof of our result, we observe that problem $\left(P_{\lambda}^{f}\right)$ has a variational structure, indeed it is the Euler-Lagrange equation of the functional $\mathcal{J}_{\lambda}$. Note that the functional $\mathcal{J}_{\lambda}$ is continuously Gâteaux differentiable in $u \in S_{0}^{1}(D)$ and one has

$$
\begin{aligned}
\left\langle\mathcal{J}_{\lambda}^{\prime}(u), \varphi\right\rangle=\frac{1}{\lambda} \int_{D}\left\langle\nabla_{\mathbb{G}} u(\xi)\right. & \left., \nabla_{\mathbb{G}} \varphi(\xi)\right\rangle d \xi \\
& -\int_{D} f(\xi, u(\xi)) \varphi(\xi) d \xi,
\end{aligned}
$$

for every $\varphi \in S_{0}^{1}(D)$. Thus, the critical points of $\mathcal{J}_{\lambda}$ are exactly the weak solutions to problem $\left(P_{\lambda}^{f}\right)$. Let $\varrho>0$ and set $\mu:=1 / 2 \lambda$, with $\lambda$ as in the statement. 
Hence, let us apply Theorem 1.2 taking $E:=S_{0}^{1}(D)$, endowed by the norm (4), $J_{\mu}:=\mathcal{J}_{\lambda}$, and setting

as well as

$$
\Phi(u):=\|u\|_{S_{0}^{1}(D)}^{2}
$$

$$
\Psi(u):=\int_{D} F(\xi, u(\xi)) d \xi
$$

for every $u \in S_{0}^{1}(D)$.

Now, $\Phi$ is sequentially weakly lower semicontinuous and coercive and $\Psi$ is sequentially weakly continuous thanks to the Rellich-Kondrachov theorem. Hence, the regularity assumptions on the functional $J_{\mu}$ are verified.

Now, we observe that there exists $u_{0} \in S_{0}^{1}(D)$ such that

$$
J_{\mu}\left(t u_{0}\right) \rightarrow-\infty
$$

as $t \rightarrow+\infty$.

Indeed, bearing in mind Remark 3.2 , it follows that

$$
\begin{aligned}
J_{\mu}\left(t u_{0}\right) & =\mu \Phi\left(t u_{0}\right)-\Psi\left(t u_{0}\right) \\
& \leq \mu t^{2} \Phi\left(u_{0}\right)-t^{\alpha} \int_{\left\{\xi \in D:\left|u_{0}(\xi)\right| \geq r_{0}\right\}} F\left(\xi, u_{0}(\xi)\right) d \xi+\kappa_{2}|D|,
\end{aligned}
$$

where

$$
\kappa_{2}:=\sup \left\{|F(\xi, t)|: \xi \in \bar{D} \text { and }|t| \leq r_{0}\right\} .
$$

Since $\alpha>2$, choosing $u_{0} \in S_{0}^{1}(D)$ such that

$$
\left|\left\{\xi \in D:\left|u_{0}(\xi)\right| \geq r_{0}\right\}\right|>0,
$$

we deduce that (15) holds.

Hence, the functional $J_{\mu}$ is unbounded from below and, by Lemma 3.4 the compactness (PS) condition is verified.

We claim that

$$
\mu>\chi(\varrho):=\inf _{u \in \mathbb{B}_{\varrho}} \frac{\sup _{v \in \mathbb{B}_{\varrho}} \int_{D} F(\xi, v(\xi)) d \xi-\int_{D} F(\xi, u(\xi)) d \xi}{\varrho-\|u\|_{S_{0}^{1}(D)}^{2}},
$$

for every $\varrho>0$. For our goal, let us fix $\varrho>0$. Since $0 \in \mathbb{B}_{\varrho}$, it follows that

$$
\chi(\varrho) \leq \frac{\sup _{v \in \mathbb{B}_{\varrho}} \int_{D} F(\xi, v(\xi)) d \xi}{\varrho} .
$$

On the other hand, it is easy to see that

$$
\frac{\sup _{v \in \mathbb{B}_{\varrho}} \int_{D} F(\xi, v(\xi)) d \xi}{\varrho} \leq \frac{c_{1}}{\sqrt{\varrho}} a_{1}+\frac{c_{q}^{q} a_{2}}{q} \varrho^{\frac{q}{2}-1} .
$$

Indeed, by $\left(f_{1}\right)$ it follows that

$$
F(\xi, t) \leq a_{1}|t|+\frac{a_{2}}{q}|t|^{q}, \quad \forall(\xi, t) \in D \times \mathbb{R} .
$$

Therefore, inequality (19) yields

$$
\int_{D} F(\xi, v(\xi)) d \xi \leq a_{1}\|v\|_{L^{1}(D)}+\frac{a_{2}}{q}\|v\|_{L^{q}(D)}^{q},
$$


for every $v \in S_{0}^{1}(D)$. Then, by (20), since

$$
\sup _{v \in \mathbb{B}_{\varrho}} \int_{D} F(\xi, v(\xi)) d \xi \leq c_{1} \sqrt{\varrho} a_{1}+\frac{c_{q}^{q} a_{2}}{q} \varrho^{\frac{q}{2}},
$$

inequality (18) immediately holds.

Since (5) holds, conditions (17) and (18) yield

$$
\chi(\varrho) \leq \frac{c_{1}}{\sqrt{\varrho}} a_{1}+\frac{c_{q}^{q} a_{2}}{q} \varrho^{\frac{q}{2}-1}<\frac{1}{2 \lambda}=\mu .
$$

Thus, inequality (16) is proved.

Then, owing to Theorem 1.2, problem $\left(P_{\lambda}^{f}\right)$ admits at least two weak solutions one of which lies in $\mathbb{B}_{\varrho}$. This completes the proof.

Remark 4.1. Theorem 3.1 can be viewed as a subelliptic counterpart of 14, Theorem 4].

Remark 4.2. We emphasize that Theorem 3.1 ensures the existence of at least two weak solutions whenever

$$
\lambda \in \Lambda:=\left(0, \frac{q}{2} \max _{\varrho>0} h(\varrho)\right),
$$

where $h:[0,+\infty) \rightarrow[0,+\infty)$ is the continuous function given by

$$
h(\varrho):=\frac{\sqrt{\varrho}}{a_{1} c_{1} q+a_{2} c_{q}^{q} \varrho^{\frac{q-1}{2}}} .
$$

Note that $\max _{\varrho>0} h(\varrho)<+\infty$, since $q>2$.

Hence, Proposition 1.1 in the Introduction is an immediate consequence of Theorem 3.1 taking into account Section 2, Moreover, we also point out that, in Theorem 1.1 . due to the presence of the parameter $\lambda$, contrary to [8, Theorem 3.1], no conditions at zero on the nonlinear term $f$ are requested.

In conclusion, we present a direct application of the main result.

Example 4.3. Let $D$ be a smooth and bounded domain of a Carnot group $\mathbb{G}$ with $\operatorname{dim}_{h} \mathbb{G}=3$. Then, owing to Theorem 3.1 , there exists an open interval $\Lambda \subset(0,+\infty)$ such that, for every $\lambda \in \Lambda$, the following problem

$$
\left\{\begin{array}{l}
-\Delta_{\mathbb{G}} u=\lambda\left(1+u^{3}\right) \text { in } D \\
\left.u\right|_{\partial D}=0,
\end{array}\right.
$$

admits at least two distinct and nontrivial weak solutions in $S_{0}^{1}(D)$. Note that in our setting, condition (1) is clearly not verified.

\section{ACKNOWLEDGEMEnts}

The authors warmly thank the anonymous referee for her/his useful and nice comments on the paper. The manuscript was realized within the auspices of the INdAM - GNAMPA Project 2015 titled Modelli ed equazioni non-locali di tipo frazionario. 


\section{REFERENCES}

[1] Giovanni Anello, A note on a problem by Ricceri on the Ambrosetti-Rabinowitz condition, Proc. Amer. Math. Soc. 135 (2007), no. 6, 1875-1879 (electronic), DOI 10.1090/S0002-993907-08674-1. MR2286099(2007k:35121)

[2] Zoltán M. Balogh and Alexandru Kristály, Lions-type compactness and Rubik actions on the Heisenberg group, Calc. Var. Partial Differential Equations 48 (2013), no. 1-2, 89-109, DOI 10.1007/s00526-012-0543-y. MR3090536

[3] A. Bonfiglioli, E. Lanconelli, and F. Uguzzoni, Stratified Lie groups and potential theory for their sub-Laplacians, Springer Monographs in Mathematics, Springer, Berlin, 2007. MR2363343(2009m:22012)

[4] Sara Bordoni, Roberta Filippucci, and Patrizia Pucci, Nonlinear elliptic inequalities with gradient terms on the Heisenberg group, Nonlinear Anal. 121 (2015), 262-279, DOI 10.1016/j.na.2015.02.012. MR3348926

[5] Haïm Brezis, Analyse fonctionnelle (French), Collection Mathématiques Appliquées pour la Maîtrise. [Collection of Applied Mathematics for the Master's Degree], Masson, Paris, 1983. Théorie et applications. [Theory and applications]. MR697382 (85a:46001)

[6] G. B. Folland, Subelliptic estimates and function spaces on nilpotent Lie groups, Ark. Mat. 13 (1975), no. 2, 161-207. MR0494315 (58 \#13215)

[7] G. B. Folland and E. M. Stein, Estimates for the $\partial_{b}$ complex and analysis on the Heisenberg group, Comm. Pure Appl. Math. 27 (1974), 429-522. MR0367477 (51 \#3719)

[8] Nicola Garofalo and Ermanno Lanconelli, Existence and nonexistence results for semilinear equations on the Heisenberg group, Indiana Univ. Math. J. 41 (1992), no. 1, 71-98, DOI 10.1512/iumj.1992.41.41005. MR.1160903 (93h:35071)

[9] Alexandru Kristály, Vicenţiu D. Rădulescu, and Csaba György Varga, Variational principles in mathematical physics, geometry, and economics: Qualitative analysis of nonlinear equations and unilateral problems, Encyclopedia of Mathematics and its Applications, vol. 136, Cambridge University Press, Cambridge, 2010. With a foreword by Jean Mawhin. MR2683404 (2011i:49003)

[10] Annunziata Loiudice, Semilinear subelliptic problems with critical growth on Carnot groups, Manuscripta Math. 124 (2007), no. 2, 247-259, DOI 10.1007/s00229-007-0119-x. MR2341788 (2008k:35066)

[11] Giovanni Molica Bisci and Vicenţiu D. Rădulescu, A characterization for elliptic problems on fractal sets, Proc. Amer. Math. Soc. 143 (2015), no. 7, 2959-2968, DOI 10.1090/S00029939-2015-12475-6. MR 3336620

[12] Andrea Pinamonti and Enrico Valdinoci, A Lewy-Stampacchia estimate for variational inequalities in the Heisenberg group, Rend. Istit. Mat. Univ. Trieste 45 (2013), 23-45. MR 3168296

[13] Patrizia Pucci and James Serrin, A mountain pass theorem, J. Differential Equations 60 (1985), no. 1, 142-149, DOI 10.1016/0022-0396(85)90125-1. MR808262 (86m:58038)

[14] Biagio Ricceri, On a classical existence theorem for nonlinear elliptic equations, Constructive, experimental, and nonlinear analysis (Limoges, 1999), CMS Conf. Proc., vol. 27, Amer. Math. Soc., Providence, RI, 2000, pp. 275-278. MR1777629 (2001h:35068)

[15] Biagio Ricceri, A general variational principle and some of its applications, J. Comput. Appl. Math. 113 (2000), no. 1-2, 401-410, DOI 10.1016/S0377-0427(99)00269-1. Fixed point theory with applications in nonlinear analysis. MR 1735837 (2001h:47114)

[16] Biagio Ricceri, Nonlinear eigenvalue problems, Handbook of nonconvex analysis and applications, Int. Press, Somerville, MA, 2010, pp. 543-595. MR2768819 (2012h:47139)

[17] Biagio Ricceri, A new existence and localization theorem for the Dirichlet problem, Dynam. Systems Appl. 22 (2013), no. 2-3, 317-324. MR3100206

Dipartimento P.A.U., Università degli Studi Mediterranea di Reggio Calabria, Salita Melissari - Feo di Vito, 89100 Reggio, Calabria, Italy

E-mail address: gmolica@unirc.it

University of Reggio Calabria and CRiOS University Bocconi of Milan, Via dei

Bianchi presso Palazzo Zani, 89127 Reggio, Calabria, Italy

E-mail address: massimiliano.ferrara@unirc.it 